\title{
Residency Training in a Healthcare Crisis
}

\author{
Hala Katato ${ }^{1} \cdot$ Daniel Smith $^{1} \cdot$ Esther Akinyemi $^{1}$ \\ Received: 23 April 2020 / Accepted: 8 September 2020 / Published online: 14 October 2020 \\ (C) Academic Psychiatry 2020
}

To the Editor:

Is the COVID-19 pandemic just a challenge or a catalyst for change?

In the medical profession, we have mastered the art of learning on the job and adapting to change. While early physicians learned through apprenticeships, medical training has progressed to more formal learning. Medical professionals have adapted to every change, starting with the plethora of knowledge expected during medical school to the daunting transition to residency. Trainees have persevered through these expected changes. COVID-19, however, is a change that was not foreseen and presents a possibility of a "new normal" in healthcare. Could it be that the COVID-19 pandemic is a catalyst for change that not only requires trainees to adapt but also the healthcare system as a whole?

Residency is the steepest learning curve for medical training. It comes with an increased patient load, longer hours, and overall greater responsibility. With the advent of COVID-19, however, we have found ourselves in an unprecedented situation. We now face a situation in which we are wedged between a deadly infectious disease, the greater responsibility of caring for these patients at the risk of death, and a yearning to grow as medical professionals while still connecting with peers. There is uncertainty about completing requirements for graduation, continuing with the medical profession, and frankly speaking, staying alive.

The fight against this novel COVID-19 pandemic has undoubtedly influenced our medical training. We have had various outbreaks and emergencies in the past that have led to alterations in traditional medical education. Though these past events may not have been as severe as our current situation, the lessons learned during those times are still valuable in the COVID-19 era.

Daniel Smith

Dsmith77@hfhs.org

1 Henry Ford Hospital/Wayne State University, Detroit, MI, USA
When SARS struck in the early 2000s, a case study from Toronto outlined the importance of maintaining regular contact between education providers and learners during the outbreak, establishing a realistic process for postponing educational courses and utilizing technological advances to deliver continued education [1]. A residency group in West Africa created a framework aligned with the Accreditation Council for Graduate Medical Education (ACGME) core competencies to help meet residency educational requirements during the Ebola outbreak in 2014-2015 [2]. This framework outlined educational goals and objectives for residents to address patient care, medical knowledge, practice-based learning, interpersonal and communication skills, and professionalism with assessments including direct observations and discussions to ensure competency [2]. HIV, SARS, EVD, and their reign in our nation have taught us how to combat deadly infectious disease whilst continuing to provide medical education.

As trainees, we quickly adapted to this healthcare crisis, perhaps more quickly than the spread of COVID-19, by taking what we have learned from historic outbreaks to further our medical knowledge and continue medical care.

The ACGME has released new guidelines that allow changes in requirements for education and graduation. This allows for institutions to declare a "Stage 3: Pandemic Emergency Status." The guidelines allow for the suspension of common program requirements and specialty specific program requirements so as to "increase the availability of physicians in clinical care settings" [3]. Ultimately, this has led to residents being redeployed to work in areas that they may have less expertise in, such as in the ICU, emergency department, or general medical floors. While this has proven to be a frightening experience for some, it has led to an increased level of comradery across specialties as they work alongside fellow trainees and attending physicians in order to provide appropriate care.

In contrast, the majority of didactics have transitioned to platforms like Zoom, potentially decreasing comradery during learning sessions. However, this could increase access to 
experts who are unable to travel and decrease time trainees spend traveling.

Another adaptation in many places is that nonemergent patient care has largely transitioned to virtual care. Virtual care previously required that the supervisor and trainee be in the same location. During COVID-19, the Center for Medicare and Medicaid Services (CMS) altered this requirement allowing the supervising physician to monitor residentpatient care through telecommunication [4].

Furthermore, CMS has allowed hospitals to count residents' work at their own home or patients' homes towards direct graduate medical education payments. For this to be in effect, residents must be practicing "within the scope of the approved residency program and meets appropriate physician supervision requirements" [4].

Additionally, telemedicine guidelines have relaxed allowing providers to utilize any nonpublic facing communication tool with patients, regardless of compliance with Health Insurance Portability and Accountability Act guidelines [5]. Lastly, there has been expansion in many states to allow physicians to provide care across state lines which was not allowed prior to this pandemic.

It is well known that a large percentage of language is nonverbal, interpretation of which is a skill critical to the psychiatric interview. With most outpatient visits being virtual, psychiatric residents have had to learn to build rapport with patients and read their nonverbal cues through a camera. Additionally, when patients return to in-person care, masks or dividers will likely be required. This presents an additional challenge for the psychiatric interview with barriers to reading nonverbal language and cues. Residency training during this time will allow psychiatric residents to be well versed in providing competent, quality care through a multitude of previously unforeseen challenges.

Many changes have occurred over the last several months that will likely forever alter how physicians practice and are trained. Through the adversity of the COVID-19 pandemic, it has become clear that these challenges are an important catalyst that can positively impact the healthcare system for years to come. Psychiatrists who have trained during this difficult time will have developed the skills to provide quality psychiatric care across multiple landscapes and barriers previously unforeseen. With the national shortage of psychiatrists, the persistence of the relaxed telemedicine and guidelines of the Health Insurance Portability and Accountability Act will prove critical to expanding access to care to psychiatrists who have specifically trained in telemedicine during this pandemic. Ultimately, trainees who have shown resilience and adaptability during this unprecedented time will progress to providing diverse psychiatric care with the ability to maneuver through any future unanticipated healthcare crises.

\section{Compliance with Ethical Standards}

Disclosure On behalf of all authors, the corresponding author states that there is no conflict of interest

\section{References}

1. Davis D, et al. Severe acute respiratory syndrome and the delivery of continuing medical education: case study from Toronto. J Contin Educ Health Prof. 2004;24(2):76-81. https://doi.org/10.1002/chp. 1340240204.

2. Mo Y, Archuleta S, Salmon S, Fisher D. Residency training at the front of the West African Ebola outbreak: adapting for a rare opportunity. PLoS Curr. 2016. https://doi.org/10.1371/currents.outbreaks. 2ccbcab30e96d3fe28d3896d258b818e.

3. Three stages of GME during the COVID-19 pandemic. ACGME Main Page. acgme.org/COVID-19/Three-stages-of-GME-duringthe-COVID-19-pandemic. 12 May 2020.

4. Teaching hospitals, teaching physicians and medical residents: CMS flexibilities to fight COVID-19. CMS. www.cms.gov/files/ document/covid-teaching-hospitals.pdf. 12 May. 2020

5. HHS Office of the Secretary, Office for Civil Rights, and Ocr. "Notification of enforcement discretion for Telehealth." HHS.gov, US Department of Health and Human Services, 30 Mar. 2020, www. hhs.gov/hipaa/for-professionals/special-topics/emergencypreparedness/notification-enforcement-discretion-telehealth/index. html. 12 May. 2020.

Publisher's Note Springer Nature remains neutral with regard to jurisdictional claims in published maps and institutional affiliations. 